\title{
Patching Logic Vulnerabilities for Web Applications using LogicPatcher
}

\author{
Maliheh Monshizadeh \\ Department of Computer \\ Science \\ University of Illinois at Chicago \\ Chicago, IL \\ mmonsh2@uic.edu
}

\author{
Prasad Naldurg \\ IBM Research India \\ Bangalore, India \\ pnaldurg@in.ibm.com
}

\author{
V. N. Venkatakrishnan \\ Department of Computer \\ Science \\ University of Illinois at Chicago \\ Chicago, IL \\ venkat@uic.edu
}

\begin{abstract}
Logic vulnerabilities are an important class of programming flaws in web applications. These vulnerabilities occur when a desired property pertaining to an application's logic does not hold along certain paths in the application's code. Many analysis tools have been developed to find logic vulnerabilities in web applications. Given a web application with logic vulnerabilities, the question is whether one can design methods to patch application code and prevent these vulnerabilities from being exploited. We answer this question by developing an approach and tool called LOGICPATCHER for patching of logic vulnerabilities. We focus on correct patch placement, i.e. identifying the precise location in code where the patch code can be introduced, based on path profiling. As we show in this paper, finding the appropriate location as well as generating the right patch can get complicated and require deep code analysis. We demonstrate the utility of LOGICPATCHER by automatically fixing several critical parameter tampering and authorization vulnerabilities in large web applications.
\end{abstract}

\section{Introduction}

Logic vulnerabilities cause a program to operate incorrectly or exhibit unexpected behavior. The ability to fix security-sensitive logic vulnerabilities in web applications, caused by incorrect control checks or improper data computation, is an important requirement in the SANS Critical Security Control (CSC) [1] for effective cyber-defenses. When such errors are exploited, they can affect the security of millions of users.

The main research issues in this context are vulnerability analysis, prevention, and correction of logic errors. While the focus of most prior research $[9,18,15,22]$ is on vulnerability analysis, and some on prevention [11], we study the problem that has received less attention, that of correcting these errors in source code. Manual inspection of the source code in order to develop security patches for reported vulnerabilities does not scale as some of these applications can be very large. As shown in this paper, however, the problem of fixing these errors automatically is far from straightforward, and requires a thorough analysis of large amount of source code. In the case of legacy web applications, if the vendor or the developer of a web application is no longer in business, or simply failed to fix the problem, the burden is on the customers of the

Permission to make digital or hard copies of all or part of this work for personal or classroom use is granted without fee provided that copies are not made or distributed for profit or commercial advantage and that copies bear this notice and the full citation on the first page. Copyrights for components of this work owned by others than the author(s) must be honored. Abstracting with credit is permitted. To copy otherwise, or republish, to post on servers or to redistribute to lists, requires prior specific permission and/or a fee. Request permissions from permissions@acm.org.

CODASPY'16, March 09 - 11, 2016, New Orleans, LA, USA

(C) 2016 Copyright held by the owner/author(s). Publication rights licensed to ACM. ISBN 978-1-4503-3935-3/16/03 . \$ \$15.00

DOI: http://dx.doi.org/10.1145/2857705.2857727 application to either change their application or patch the vulnerabilities themselves. Patching an application requires a thorough understanding of the application and the error, something that deployment professionals do not have time for.

\subsection{Logic Vulnerabilities}

In the web applications context, logic vulnerabilities may manifest in one of two categories: 1) as a logic error in a computation (data dependent) or 2) as a logic error in program control, i.e., a vulnerability caused by a missing or an incorrect control check in the program. Web applications often do not come with correctness specifications - properties that attest behavior at various program points. The lack of such program specifications makes it very difficult to identify original functional requirements and validate their correctness. Therefore, fixing the first category, i.e., computation errors is challenging, as the true intention of the computation is unknown to the analysis tools. However, the second category of logic vulnerabilities is relatively easier to generate patches for, given that this subset of logic vulnerabilities are detectable through inconsistency analyses on applications. We term this set of vulnerabilities as application inconsistency vulnerabilities (AIVs), the type of logic vulnerability that arises from inconsistent design and implementation of security checks in an application. Existing tools for AIVs $[18,15]$ can not only detect that something is missing or is incorrect, but also infer what exactly is missing and suggest ways to correct it.

In addition to generating correct conditions for these checks, the correct placement of these is also a difficult problem. Detected vulnerabilities may be embedded deeply in the code, beyond such easily analyzable or stand-alone constructs such as user interfaces or end-user communication modules. Manually locating these errors may require rigorous analysis of the source-code, with many interdependencies. Modifying or adding code snippets to fix these errors will now imply we take extra care not to change the logic and the functionality of the interdependent code, and only fix the vulnerable path(s). To address this, we have designed and implemented LOGICPATCHER, a static analysis tool that takes a patch condition, i.e., a reported vulnerability and a path descriptor as input, and suggests optimal or near-optimal candidate path placement locations. Using our automated tool, application customers or system administrators can confirm and test the candidate patches instead of going through the arduous work of manual code inspection.

Due to fundamental and practical issues in verification of code correctness, the candidate patches need to be verified by a separate approach, either manually or automatically through formal verification. For reasons of scope, we discuss the issues of patch generation and patch placement for AIVs in this paper, and leave the formal verification step for future work. Additionally, we have employed several static code analysis techniques which increase our confidence in the correctness of the candidate patches. We evalu- 
ated 9 open-source web applications and out of 29 vulnerable files we have generated patches for 27 of them correctly.

\subsection{Main issues}

At a high level, we need to identify the candidate patch locations for the application while preserving the logic of the program. This goal becomes challenging as we do not know much about the functionality of the program in the first place. The only information we have aside from the source code of the application, is the reported vulnerabilities and their locations in the code.

Patch Generation: In order for the generated patch to work, it should first have the necessary instructions which will prevent the exploits. While the generated patch should work, it should not interfere with the main functionality of the program.

Patch Placement: We need to find the proper location for the patch, which assures that the patch will not change the logic or the functionality of the application along other execution paths. For patch generation, we rely on outputs from AIV detection tools such as Rolecast and MACE [18, 15]. Our work in patch placement is related to FixMeUp [19], a static analysis tool that suggests patches for access control vulnerabilities. Our research finds a wider scope for solving this problem. We believe that we can generate security patches for any type of logic vulnerability caused by missing or inconsistent checks, with minimal guidelines about the vulnerability, and find optimal or near optimal placement of the suggested changes directly. We summarize the contributions of our research: 1) Precise formulation of logic vulnerabilities in order to patch the applications, 2) Design and implementation of an analysis tool called LogicPatcher, 3) Finding candidate placements of the generated patches along the vulnerable path(s), and 4) Generation of security patches for reported logic vulnerabilities for 9 open-source applications.

This paper is organized as follows: Section 2 presents a running example used in the rest of this paper. Section 3 explains the high-level overview of our approach. Section 4 describes the architecture of LOGICPATCHER and the implementation details. Section 5 presents the evaluation of our approach. Section 6 presents related work. In Section 7 we conclude.

\section{Background and Challenges}

Application Inconsistency Vulnerabilities (AIVs) are cause by lack of consistency in the design / implementation of security checks. Some of them include:

- E-commerce logic inconsistencies These vulnerabilities result from inconsistent checking of business validation logic in the application code. Prior work on detecting these vulnerabilities include using model checking [9], modeling correct payment logics combined with static analysis [20], and invariant generation and blackbox testing [16]. In all these cases, the vulnerability analysis tools report inconsistencies in checks, and these inconsistencies are subsequently verified for the presence of vulnerabilities.

- Client-server inconsistencies The validation performed by clientside JavaScript can be used as a specification to check the serverside for vulnerabilities. $[6,7,3]$ take this approach towards vulnerability detection. In this case, the vulnerability is a client-side check that must have been performed by the server.

- Access control inconsistencies Inconsistency in application authorization logic along different execution paths result in application vulnerabilities. $[18,15,22,10]$ look for these types of inconsistencies, which are subsequently confirmed for the presence of actual vulnerabilities.

\subsection{Vulnerabilities to Be Patched}

AIVs seen in Table 1 often have one characteristic in common: they lack conditions (if-statements) in one or more execution paths.
Table 1: Inconsistency Checking Analysis Tools

\begin{tabular}{|l|l|}
\hline Tool & Description \\
\hline Waler [9] & $\begin{array}{l}\text { Detection of program invariable violations } \\
\text { through model checking }\end{array}$ \\
\hline JIGSAW [23] & Detection of resource access inconsistencies \\
\hline MACE [15] & $\begin{array}{l}\text { Detection of authorization \& authentication in- } \\
\text { consistencies }\end{array}$ \\
\hline RoleCast [18] & $\begin{array}{l}\text { Detection of authorization \& other logic incon- } \\
\text { sistencies within authorization roles }\end{array}$ \\
\hline WAPTEC [7] & $\begin{array}{l}\text { Detection of client and server input validation in- } \\
\text { consistencies }\end{array}$ \\
\hline
\end{tabular}

That is, some conditions are not met along some paths. This problem makes these paths and eventually the whole application vulnerable to various exploits.

To patch this type of vulnerability, the developer needs to have some basic knowledge about the vulnerability to be able to patch it effectively. In particular three different items affect the patching process: $\langle C, P, E\rangle$ in which $C$ is the missing condition which needs to be added to vulnerable paths, $P$ is the particular vulnerable path, and $E$ is the exception handling policy the developer considers if the condition failed at execution time.

The Conditions Set The set of missing conditions $C$ is expressed in terms of variables, values and conditional operators. For example, $\{$ strlen (\$password) $, 8,>=\}$ defines a condition on the length of the variable \$password. The variables used in $C$ can be functions of input variables (e.g. strlen (\$password)) or they can be internal variables related to server-state (e.g. \$_SESSION [' username' ] ). The values also can be constants or derived from some server-state (e.g. the result of a DB query).

The Vulnerable Path(s) $P$ is the path from the source to the sensitive operation $(\operatorname{sink})$. Security analysis tools can usually generate execution traces which shows the possibility of the exploiting the vulnerability.

The Exception Handling Policy Set The set $E$ defines the set of actions which are allowed to be executed if the conditions $C$ do not hold. For example, the developer may choose to use exit () or die ("message") or she may choose to log the failed operation in the system. The semantics of the actions specified in $E$ depends on the application, the usage and developer's choice.

\subsection{Goals \& High-level Challenges}

Our high-level goal is: given a inconsistency logic vulnerability, we want to generate a candidate patch to retrofit the vulnerable sink. To generate such security patch analyses must be performed to 1) generate a proper patch and 2) place the patch in a proper location.

There are some high-level challenges involved in the process of generating security patches for vulnerable web applications. Given that most web applications lack program specifications, our approach should be able to work on the source code with minimal input from the developers/application admins. That is, the patch generation and placement modules should rely on the extracted logic of program and inferred security policies.

Missing Check Dependencies A security patch is basically a generated code snippet which is going to be placed somewhere along the vulnerable path(s). The code snippet may contain variables which should have semantical and syntactic values at the location they are going to be placed. Therefore, the data dependencies for these variables should be kept intact and meaningful. Forward and backward data dependencies of the variables used in the code snippet will assure that the dependencies are correct and consistent along different paths and throughout the application.

The following example, derived from a real-world vulnerable application, shows a sink (the DELETE query) in which the variables 
used in the query are dependent on some previous instructions. Although the value used in the sink depends on the user input (e.g. \$_POST ['username']), it also depends on some server state (e.g. the data stored in users table which determines if the username is valid in the application). If the sink missed the authentication, along with the check in Line 2, other instructions (on validation of the username and password) should accompany the check in the patch. Therefore, we need analysis to determine the instructions with dependencies on the input values and the server-state.

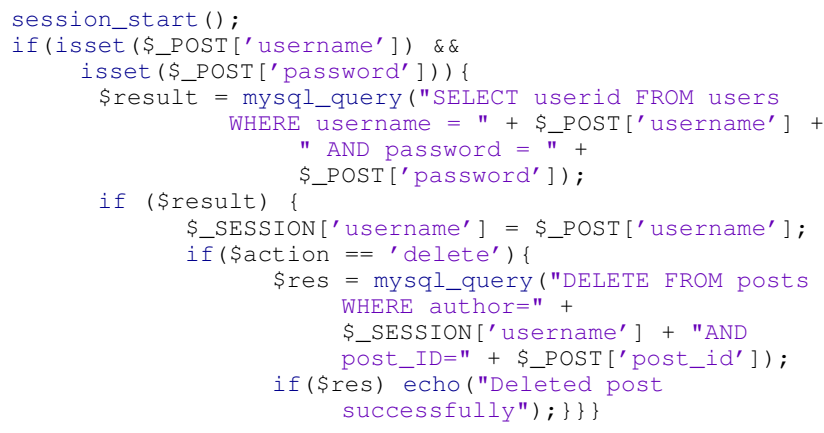

Listing 1: Sink Control and Data Dependency

Overprotecting The patching process includes adding some code snippets (patches) to the source code which will retrofit certain vulnerable sinks. Therefore, we need to make sure that the changes made to the application source code do not in any way affect the functionality and logic of the program in the execution paths which were not vulnerable. In particular, we need to assure that the logic of the program in other non-vulnerable paths is not changed due to the insertion of the patch. The path-sensitivity of the patching problem requires us to know which path it is going to retrofit. Our analysis can gain this information about the vulnerable path from the vulnerable location (sink) and can use this information to distinguish execution paths.

Optimization Although the main goal of automatic retrofitting of the web application against logic vulnerabilities is to make sure that the vulnerabilities are correctly patched, but we also prefer that the resulting web application source remains optimized in terms of both time and space. That is, we prefer to add one patch at a location where it prevents several vulnerable locations than adding multiple patches into multiple locations in the code. To place the patches to optimal locations in an application, our approach considers various scenarios with different candidate places, it then identifies which candidate place is optimal for an application with one or multiple vulnerable sinks. More detailed discussion about optimization of the patching process is in Subsection 3.4.

\section{Approach}

Figure 1 shows the overview of our approach. There are two two main steps: 1) generate the appropriate patch, 2) place the patch in an appropriate location in the original code.

Input Our approach uses general information about the vulnerability to start its analysis. This information includes: 1) the missing condition, $C, 2$ ) the path to the vulnerable sensitive operation $(\operatorname{sink})$, $P$, and 3 ) the exception policy $E$.

Error Handling Policy LogicPatcher requires an (optional) exception handling logic $E$ as input. If no inputs are provided, the default exception handling strategy is to terminate of the program. This way if the placed security condition $C$ is not met by the program state, the program terminates without entering in a non-secure state. Though termination is one option, the developers may want to take other actions if a malicious input is given to the program. Logging and sending alerts to system administrators are among popular actions that one may take after a malicious attack. To better help users of the tool in deciding the exception handling methods, LOGICPATCHER provides an option to analyze exception handling options in the source code (see Appendix A).

\subsection{Patch Generation}

The Missing Conditions Automatic detection tools usually report AIVs in terms of the missing conditions as well as the details about the location of the vulnerability. They express these conditions in terms of conditions on 1) user inputs or 2) conditions on the internal server state. A condition is a tuple of $\langle\mathrm{Var}, \mathrm{Val}, \mathrm{Rel}\rangle$ in which Var is the variable, Val is the value for the variable which may be a constant value or a dynamic one, and Rel is the relational operator. Our approach can use the missing conditions $C$ and the vulnerable execution trace (path) $P$ to compute the program slice for these constraints. This program slice is called the patch.

The security conditions to be added to the patch include variables which specify the available information context. This information context defines the type of the patch as well as the instructions which need to be added to patch.

Program Slicing A missing condition, when inserted in code, may need to be accompanied by some other data- and control-dependent instructions if necessary. To preserve the data dependencies, we may add other instructions to the patch. That is, given a condition $<$ Var, $V a l$, Rel $>$, we perform backward program slicing so that the variables Var and values $\mathrm{Val}$ involved in the condition set $C$ will be meaningful and valid at the patch location.

\subsection{Patch Placement}

The problem of patch placement may seem to be trivial at first glance. However, the location of the vulnerability is not necessarily the location where the security patch should be placed. A logic vulnerability patch is basically a constraint written as an conditional statement. To insert an conditional statement we need to find the starting point and the ending point for the conditional statement block.

An important challenge in patching an execution paths is to ensure that the patch does not affect other execution paths, an occurrence we call overprotection, since adding a security condition to a path which was not vulnerable may make that execution path unavailable, and prevent it from being executed under legitimate circumstances. To place the generated patch, the approach should find candidate places in the code where it can insert the beginning and ending of the conditional statement block without interfering with other execution paths and other sinks.

Multiple Paths to one Vulnerable Sink When only some of the execution paths to a sink are vulnerable, we need to make sure that we are fixing those vulnerable paths only and are not changing other paths. When there are multiple execution paths to a vulnerable sink, our approach must assure that the vulnerable(s) path is patched, as well as ensure other non-vulnerable paths remain intact. Based on this goal, we categorize different scenarios when placing the patch.

To show these scenarios, we provide an example of a sink (a DELETE query). The code example deletes some records from the table $\$$ table. This example derived from a real-world example in phpNS application (simplified for more clarity). The main command to delete is executed in function.php.

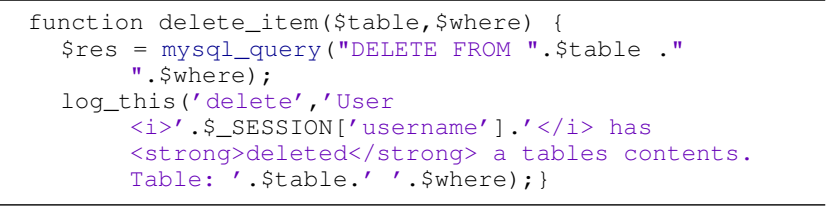

Listing 2: Delete Query phpNS, function.php 


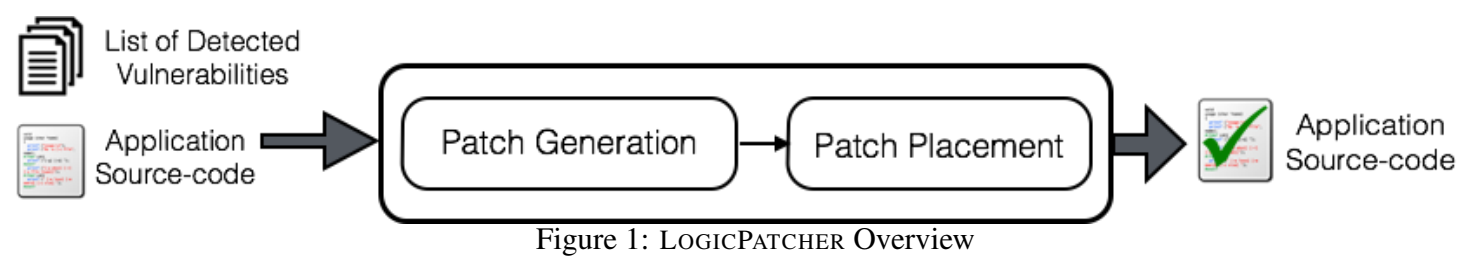

Figure 1: LOGICPATCHER Overview

Analysis shows that this query is vulnerable to authorization bypass in some of the paths, for instance it lets the authenticated normal users to delete any comment in the system if they can guess the comment ID (which is an incremental integer value and not hard to guess). The missing condition is to check whether the current user is the owner (the author) of the comments and if so, she can delete the comments. Now let us consider different scenarios where the other execution paths in the application affect our decision about patch placement. For code in Listing 3, all of the paths leading to the sink are vulnerable since both paths allow authenticated users to delete any comments.

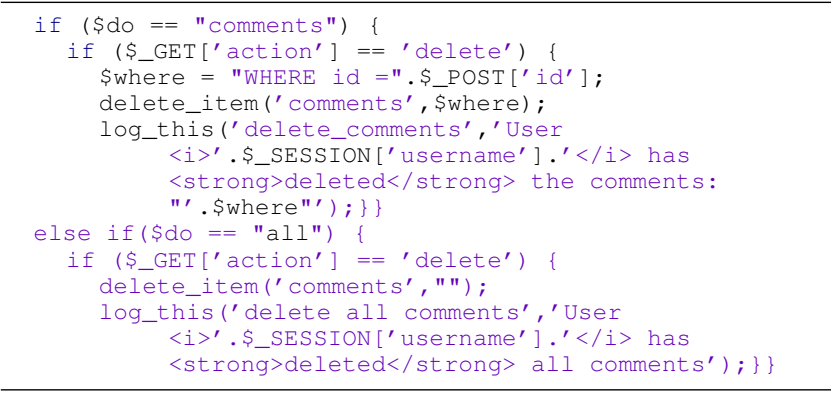

Listing 3: Deleting a comment, article.php
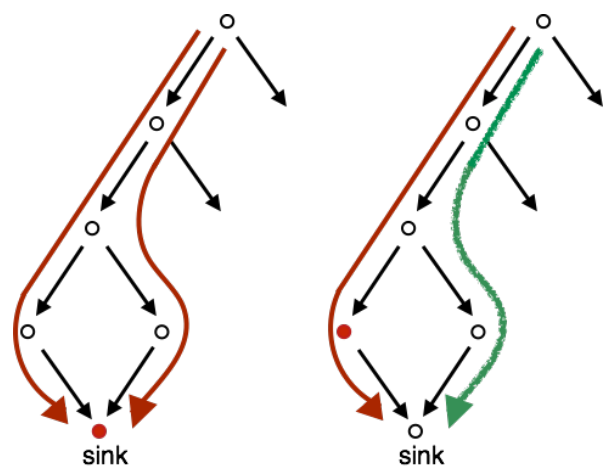

Figure 2: a) Execution paths for Listing 3, b) Execution paths for Listing 5

An abstract control flow graph of this example is depicted in Figure 2 (a) where both paths to the sink are vulnerable and therefore we can simply place the patch at the sink location. Since all of the paths to a sink are vulnerable, then patching the query or patching the path to the query is straightforward: it can insert the patch just before sink location. We can place the patch, which is a check for the author of the comment (users should be able to delete their own comments), in the delete_item function if this function is used only for deleting comments. Listing 4 shows the candidate place for the patch.

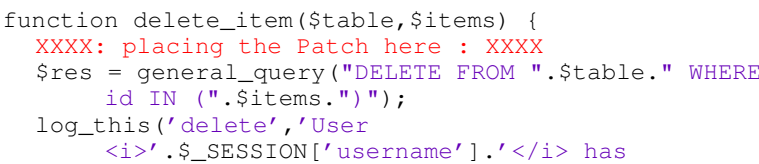

<strong>deleted</strong> a tables contents. Table: '.\$table.'. Items: '.\$items);

Listing 4: Patching scenario 1, phpNS, function.php

Another scenario is shown in Listing 5 in which only some of the paths to the query are vulnerable. The path to deleting a comment is the vulnerable one but the other path to delete an article is not since it restricts the query to the author of the article.

Figure 2 (b) shows the control flow representation of Listing 5. If we place the patch inside delete_item function, then we are adding code to non-vulnerable paths, in which we will check for the author of comments, and so we are injecting an irrelevant check to the code which makes the query unavailable for those nonvulnerable paths.

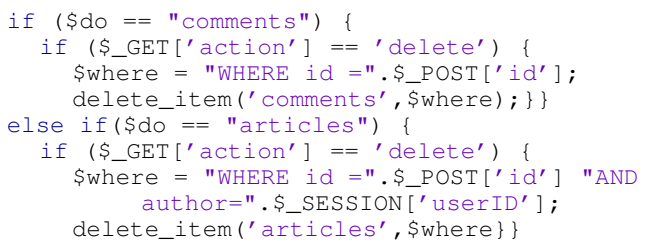

Listing 5: Non-vulnerable Paths to the Sink, article.php, phpNs application

Unlike the earlier scenario, in these case, we certainly cannot patch the path at query location. To patch the vulnerable path, we must ensure that the patch is only accessible along the vulnerable path. Our approach finds the nearest location from the sink where the patch would not interfere with other paths. The candidate location for this example is shown in Listing 6 .

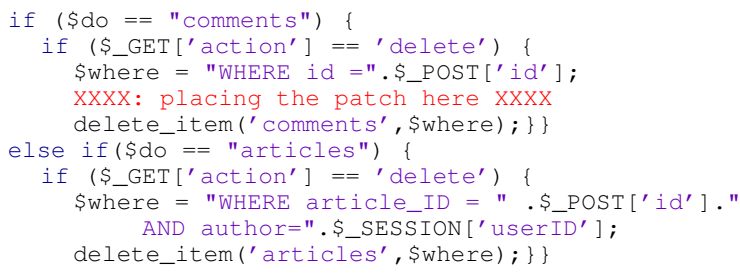

Listing 6: Patching scenario 2, phpNS, article.php

Other Paths to Other Sinks Now consider a scenario in which some paths to the sink are vulnerable, but the vulnerable paths are not entirely disjoint from other non-vulnerable paths to other sinks. Listing 7 shows the example where the code is shared with other users in the system (in this case admin user(s)). Based on the previous solution, we may place the patch before calling function delete_item, however, we are restricting the admin users from deleting comments of other users, where there is no such policy in the application demanding that.

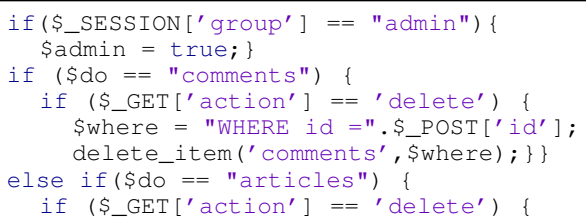


delete_item('articles', "WHERE article_ID = "
.\$_POST['id']." AND

author=".\$_SESSION ['userID']); \}

\section{Listing 7: Other Execution Paths in article.php}

Figure 3 (a) shows this scenario. Although the nearest node to sink 1 is node $x$, we cannot place the the patch right after this node, because it will interfere with the non-vulnerable path.
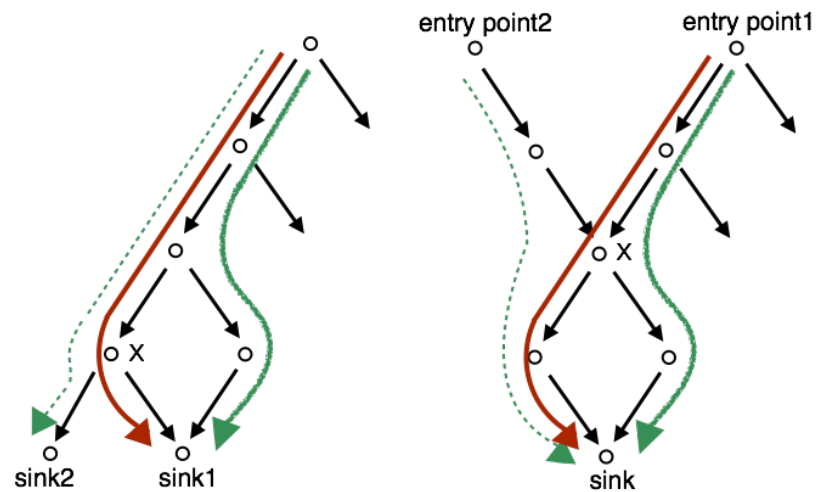

Figure 3: a) Interference with Other Execution Paths: CFG representation of Listing 7, b) Interfering Execution Paths: Non-disjoint Paths

The above example, or even the one shown in Figure 3 (b)), where two or more non-vulnerable paths are not entirely disjoint from the vulnerable path, show that in some cases the overlap between the paths make it impossible to place the patch along the vulnerable path without interfering with non-vulnerable ones.

In order to uniquely patch the vulnerable path, we need to be able to uniquely identify it. Therefore, our approach uses Path Profiling techniques [5] to uniquely identify the execution paths in the instrumented application. Path Profiling algorithm assigns a unique state to each execution path and instruments the program with state transitions on each path. The changes in the transition state should be in a way that by reaching the end of each path, we reach the same state which was assigned to the path. Path Profiling algorithm introduced in [5] computes the state transitions efficiently.

After identifying the path, and checking for interfering paths, if inserting the condition $C$ alone results in interference between vulnerable and non-vulnerable paths, then the path id (hash ids generated in path profiling) will be merged with condition $C$, to make it non-interfering, as shown in Listing 8.

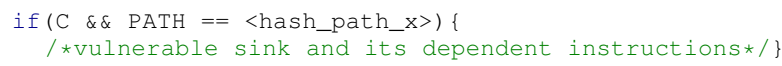

\section{Listing 8: Merging Path Profiling info}

\subsection{Algorithm}

A high-level overview of our algorithm is shown in Algorithm 1. We start our analysis by parsing and generating the controlflow graph (CFG) of the source code. After creating the CFG, we should identify the CFGNodes which are data- or controldependent on the sink. This is a crucial step since we are going to wrap the sink inside an introduced security condition $C$; therefore we need to also include these dependent instructions inside the condition block so that the program executes consistently during run-time. Sets instructions_before_sink and instructions_after_sink are used to gather these dependent instructions.

Finding the right Scope The previous scenarios show that it is important to reason about the patch and the placement of the patch

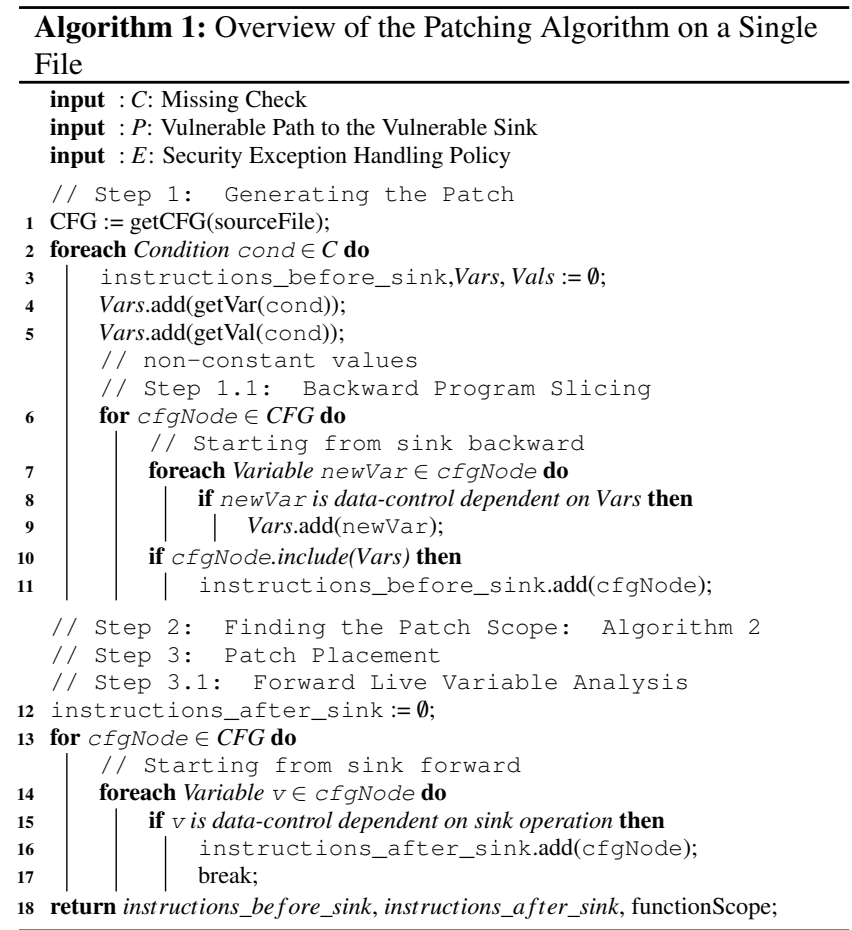

with respect to other execution paths in the application.Our approach uses static analysis techniques to generate and place the patch in a proper location and it also reasons about the possibility of multiple vulnerabilities and how LOGICPATCHER should generate and place the patches so that they would also not interfere with each other.

Recall that the input to LOGICPATCHER is a vulnerable sink location $P$, the missing (authorization) condition $C$, and the security exception handling policy $E$. LOGICPATCHER generate 1 ) the patch $S$ which is a set of instructions; 2 ) the candidate location $L$ for the patch to be inserted in. $L$ consists of the patch context (i.e. filename where the patch introduced), and two candidate locations to insert the start and ending of the missing condition.

First our approach needs to identify the function context where we should inject the missing condition. Since the execution paths are intra- and inter-procedural according to where in path we inject the missing condition, LOGICPATCHER may work with different function contexts.

Consider this sequence of function calls $f()->g()->h()$ where function fcalls function $g$ and also function $g$ can call function $h$ and function $h$ includes the vulnerable sink. The first challenge is identify the proper function context; that is, whether LoGICPATCHER should add the missing condition to function $\mathrm{h}(), \mathrm{g}($ ) or $f()$.

To answer this question we should think about our second goal: not to overprotect the application, which means that we should not add the condition to the execution paths which were not vulnerable in the first place. So we first examine the number of function calls to each of this functions - starting from the innermost function $h()$ and going backward to $f()-$ and if the sequence of such function calls includes paths which are not vulnerable we go one step backward.

After this step, the location of the function is found and the scope of the variables involved in the patch is identified. Now LogICPATCHER needs to find the start and end block for the condition itself. The start point for the condition can come just before the vulnerable sink inside the candidate function $\mathrm{f}$. 


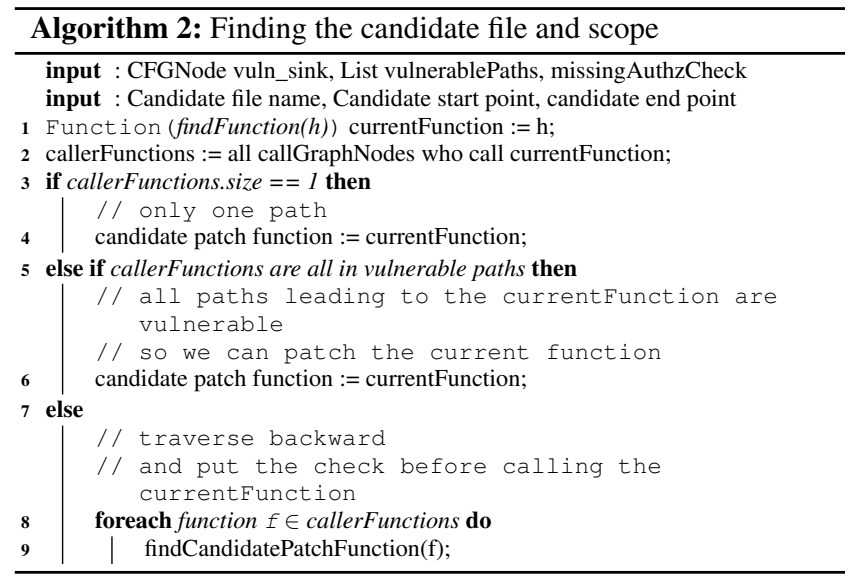

For the end point of the check, we first need to analyze the data dependencies after the sink. To address this problem, we do an inter-procedural Live Variable Analysis (LVA) on the output of the sink operation to find the scope of the code where the output is still live. This analysis may add other variables to the set due to data dependencies.

Live Variable Analysis A patch puts the vulnerable sink inside an conditional statement block with the condition $C$. If we simply put only the sink inside a conditional statement, we may change the original semantics of the application. Merely including the sink instruction to the conditional statement block may make other instructions after the sink invalid. Listing 9 shows an example where the result of the query is used to other operations.

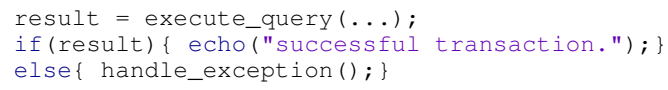

Listing 9: Result of sink is used for another operation

Now one only puts the sink (the query) inside the condition block, then the result value would not be accessible for paths which do not execute the true branch of condition $C$. Therefore, our approach needs to reason about a candidate location for ending the condition block.

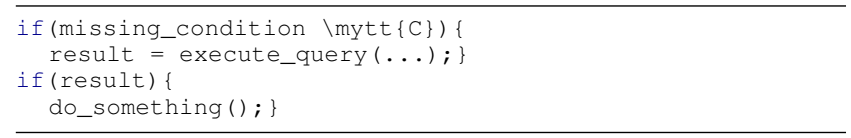

\section{Listing 10: Wrongly patched version of 9}

The correct patch for Listing 9 is shown in Listing 11.

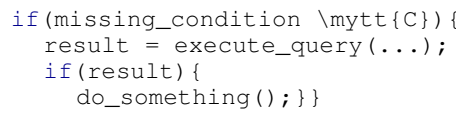

Listing 11: The correct patch for 9

What is changed in Listing 11 is that every instruction which is control- or data-dependent on the value of the sink operation is now also contained by the condition block. To find all of the instructions which are dependent on the sink value, we should first find the variables which are dependent on the value of the sink. Each instruction which includes such variables, will be added to the instruction list which is going to be added to the condition block.

To find the sink-dependent variables LOGICPATCHER performs forward data-control dependency analysis starting from the sink location and put the ending of the block where the result of the sink is no longer used. LOGICPATCHER uses similar ideas for Live Variable Analysis to find which variables are dependent on the sink. The implementation details of this algorithm is shown in the implementation Section.

\subsection{Discussion}

Patching the Sink Based on the type of vulnerability and the information context available in the execution paths, there may be two options to patch the application at the source level: 1) patching the

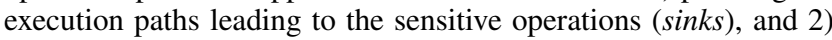
patching the sink itself by changing the access parameters of the sensitive operation.

For example, when the sink type is a DB query, to patch the vulnerability we may be able to augment the query where clause if the columns in the DB table hold the condition variables. This way the query becomes more restrictive. However, it is not always the case that the values are available for access parameters. For example, when the missing authorization information is not available in the query or in any of the columns of the table, we need to add the missing conditions to the execution paths leading to the vulnerable sink.

As an example, consider the following query. This query is accessible for all logged-in users of a blog application. The following query is detected as vulnerable because it lets the authenticated user to delete any blog post if she can provide a valid post ID.

\$SqI = "DELETE FROM blogdata WHERE post_id=\$post_id";

\section{Listing 12: Query to be Patched}

To patch the vulnerability, we may have the option to place the condition authorID == \$_SESSION [ 'userID' ] in the WHERE clause in the query as shown in Listing 13 if the blogdata table contains the column author_ID. Alternatively, we can place the condition in the path leading to the query as shown in Listing 14.

$$
\begin{aligned}
& \begin{array}{c}
\text { \$SqI = "DELETE FROM blogdata WHERE post_id=\$post_id AND } \\
\text { author_ID=\$author_id"; }
\end{array} \\
& \text { Listing 13: Patched Query } \\
& \hline \begin{array}{c}
\text { if(getAuthor(\$post_id) }==\text { \$author_id | | } \\
\text { \$author_id=="admin") }\{ \\
\text { \$SqI = "DELETE FROM blogdata WHERE post_id=\$post_id"; }
\end{array}
\end{aligned}
$$

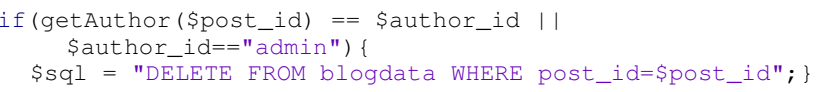

Listing 14: Patched Path

Patching Multiple Vulnerabilities In real world, it is often the case that an application may have more than one logic vulnerability. It is because some logic vulnerabilities go together i.e. they may have the same or similar root cause(s). In patching logic vulnerabilities therefore we should examine how we can patch the application in a way that is both 1) correct and 2) efficient. Reasoning about patching multiple vulnerabilities is crucial because we do not want to repeat the patch(es) firstly because it may change the logic of the program and secondly because repeating the patch at multiple locations is not time- and space-efficient. In finding the optimal location for patching multiple vulnerabilities in an application several scenarios may occur:

1) Vulnerabilities of the same type: A patch consists some security checks which would control values on some variables or some server-side state. Vulnerabilities of the same type may share these checks entirely or partially. In this scenario, we should place the checks if they are being added previously.

2) Vulnerabilities with various types, but the same root cause: In another scenario, the vulnerabilities may be of various type and therefore they do not share the security checks in the patch, however, they share a common prefix in the code. i.e., they share paths.

In this case, even though the checks themselves are not shared, but the patch may change data-dependencies for the variables used in the patch or they may change the server-side state. Hence, we 
must watch out for the instructions included in each patch to make sure they do not interfere with each other.

3) Independent Vulnerabilities: Vulnerabilities are independent when they do not share a common prefix in the code or they do not have the same cause. These vulnerabilities can be patch independently. Our approach takes each vulnerability one by one and generated the candidate patch for it.

\subsection{Limitations}

LOgicPatcher is a 'best effort' tool which suggest security patches to developers/system administrators. A separate formal verification or manual effort must be made to verify the correctness of the generated patches and the resulting source code. This is because of some high-level limitations which are involved in using automated tools to patch security vulnerabilities which we discuss in the rest of this subsection.

Lack of Program Specification The main reason that our approach cannot assure the correctness of the patches is that our approach does not require specifications on the functionality of the program, functions and program statements in the source code. For instance, during the patch generation, our tool must decide which instructions should be wrapped by the security condition $C$. This would be much easier if the tool had more knowledge about the sensitive instructions rather than performing static data- and controldependency analyses.

Although we do not verify the correctness of the patches automatically, we believe that the proposed approach by design minimizes undesirable side-effects on the functionality of the applications. For instance, in the presence of interfering execution paths, as discussed in Section 3, we use path profiling to prevent the introduction of new control instructions in non-vulnerable paths.

The generated patch consists of the missing condition, and its control and data dependencies. Thus, the correctness of the generated patch depends on the correctness of the data and control dependency analysis. We use backward program slicing and live variable analysis techniques to create the patch. These data and control dependency techniques are performed statically and therefore they may be imprecise. However, we use a very conservative approach to analyzing the dependencies along the execution paths and so we do not miss any dependent instructions. For instance, in backward program slicing, even though the dependencies of DB query instructions are not available statically, our tool adds those instructions in the patch if condition $C$ is dependent on the result of the query and also it adds the query string variables to the set of variables of interest. This may seem to cause overprotection problems, however, the path profiling procedures ensure that the instructions protected by $C$ are going to be used only in the vulnerable path.

Cascading Sinks There are some complications involved with patching applications in which the developers use cascading sinks. Assume that the developers perform two sensitive operations (reading and writing to $\mathrm{db}$ tables, or files) in the same execution path, and one of these sensitive operations is vulnerable. The decision to whether include both sinks in the missing condition statement block or just include the vulnerable sink depends on whether the data- and control-dependency analyses detect the two operations as related or not. The problem may cause some side effects (as we will see in Section 5 under correctness discussion) on the generated patch and correctness of the code.

\section{Implementation}

We have designed and implemented a tool which, given a list of application vulnerabilities in a web application, it will generate security patches for the vulnerabilities and will place them in the source-code of the application so that each sink is no longer vulner- able to that particular vulnerability. Figure 4 shows the architecture of LOGICPATCHER in two major steps: 1) patch generation and 2) patch placement. As shown in this figure, each phase has various analysis components. In this section we go over the implementation of each of these components.

\subsection{Inputs to LOGICPATCHER}

LOGICPATCHER starts the analysis with two sets of inputs:(1) source files and (2) list of detected vulnerabilities which is expressed as the tuple $\langle C, P, E\rangle$ (ref Sec 2.1). In our experience, providing such input does not need any additional effort. Security tools usually report the vulnerabilities with these details.

\subsection{Patch Generation}

To generate the security patch, LOGICPATCHER uses static dataand control-flow analysis to preserve the original semantics of the program in the presence of a new security condition $C$. A security condition has a set of variables and values which should be have the same semantics as other consistent paths while used in the security condition. Therefore LOGICPATCHER uses static analysis to identify the instructions which include these variables and values. These instruction then are then added to the patch.

LOGICPATCHER starts with using a PHP parser and Control Flow Graph (CFG) generator to get the CFG of the application. It then traverses the CFG of one of the consistent paths backward from the sink location to find the related instructions to condition $C$ 's variables and values. This analysis is intra- and inter-procedural which enables LOGICPATCHER to reason about consistency of the patch throughout the entire application. After adding all the related instructions to the patch, LOGICPATCHER compares these instructions to the instructions which are in the vulnerable path $P$. The reason for this comparison is that our approach avoids the insertion of instructions to a path twice as it may cause side-effects.

\subsection{Patch Placement}

As discussed in Section 3, along the execution path leading to the vulnerable query, there are several candidate locations to put the patch. If we put the patch at the beginning of the entry point, we may cause so many side-effects because other instructions are going to be affected by the patch. So the best choice is to put it as close as possible to the query itself to minimize the side-effects.

To find a correct place for the patch to be inserted in the code, LogicPatcher uses several code analysis techniques. These techniques assure that LOGICPATCHER inserts a patch into the vulnerable path and it does not affect other execution paths.

Path Enumeration To enumerate all of the execution paths, LoGICPATCHER uses Pixy [13] to parse all of the PHP files in the source code and build the Control Flow Graph (CFG) of the application. LOGICPATChER then traverses the CFG nodes in the graph and lists all of the paths for each entry point. It finally gathers all of the execution paths for all of the entry points in the application. Our path enumeration is intra- and inter-procedural.

LOGICPATCHER starts uses one entry point at a time to enumerate all of the paths starting from that entry point. For non-control instructions LOGICPATCHER adds the CFG node for the instruction to the current path to be constructed. Each time LOGICPATCHER visits a control instruction (i.e. if-statement, function call, function return) it changes the list which holds the paths.

For if-statements it clones a path into two different paths and puts the paths back into the list of paths. For a function call instruction, LOGICPATCHER first saves the return address (the next instruction to be executed after the function return) in a hash structure and then traverses the CFG for the function. By visiting a function return, LOGicPATcher searches the hash and adds the CFG node for the return address to the current path.

Path Profiling As discussed in Listing 5, there may be cases where 


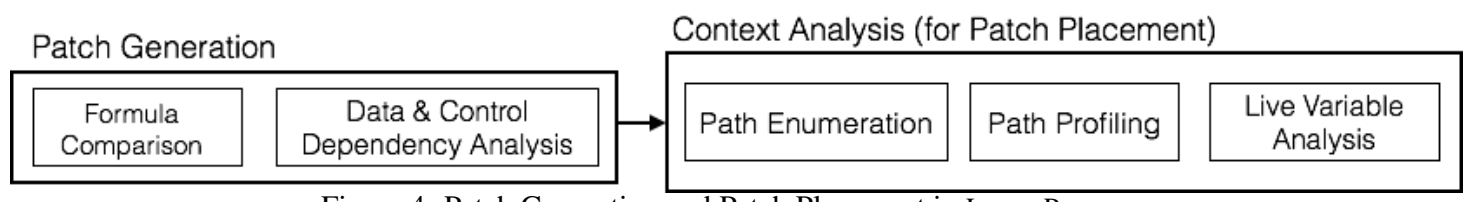

Figure 4: Patch Generation and Patch Placement in LOGICPATCHER

placement of the patch in a vulnerable path interferes with other execution paths since it is not disjoint from the other paths and therefor we may need to introduce extra control flags to assure that the patch is going to be added to the vulnerable path only. In order to patch the vulnerable path and only the vulnerable path, LoGICPATCHER needs to keep track of the vulnerable path and uses some criteria to uniquely identify it.

Path profiling techniques [5] enable LOGICPATCHER to achieve this goal. The path profiling concept first enumerates all of the paths and assigns a unique number to paths to each sink or exit location. It then computes the transition numbers and instruments the application. At execution time, by each transition, the computed value for the transition is going be added to the state of application. Eventually when it reaches the sink or exit location it arrives to the same assigned number to the path. LOGICPATCHER uses the same ideas to keep track of the vulnerable path and for efficiency it uses an abstracted version of the path which only includes controlinstructions: conditions, loops, exit, return, and function calls.

Live Variable Analysis As discussed in Section 3, there are instructions after the sink which need to be included in the condition block during the patching. Since we need to know the last location where the dependent variables are used (the result value of the sink operation is still alive), we need to perform a backward dependency analysis, which is going to show the last location where the variables are used. LOGICPATCHER uses Live Variable Analysis (LVA) [2] ideas to keep track of the variables which are data- or control-dependent on the result of the sink operation.

To perform the analysis, LVA algorithm takes the current context (i.e. the current file which the patch is going to be inserted in) as input and performs a backward analysis to compute the In, Out, Def and Used sets. The last instruction where all sink-dependent variables are still alive is going to be marked. The end of the condition block will be just after the marked instruction. Since the value of the sink operation may be used in return statements and be variables in other file contexts may be dependent on the sink, LoGICPATCHER performs intra- and inter-procedural LVA. Note that the end block will be inserted to the file context where the condition is going to be added, therefore the block syntax is preserved by LOGICPATCHER.

Output LogicPatcher generates a candidate security patch which includes the missing condition $C$. It also gives out the candidate location to place the patch which is somewhere along path $P$. In cases where path profiling is needed, LOGICPATCHER produces an instrumented version of the code along with new control conditions, which assure that the patch is only executable along the vulnerable path.

\subsection{Discussion}

There are some technical limitations associated with static analysis of programs for patching PHP applications with current opensource tools. These problems however are not derived from the design of LOGICPATCHER, and they do not limit the applicability of our approach for patching applications.

Object-Oriented PHP features LogicPatcher uses Pixy [13] for parsing and creating the control flow graph of the application. Some complex features in PHP such as object-oriented features are not handled by Pixy.
Table 2: PHP Applications

\begin{tabular}{|l|l|l|l|l|l|}
\hline Application & SLOC & $\begin{array}{l}\text { \# php } \\
\text { Files }\end{array}$ & $\begin{array}{l}\text { \# of Sink } \\
\text { Locs }\end{array}$ & $\begin{array}{l}\text { \# of } \\
\text { Resources }\end{array}$ & $\begin{array}{l}\text { Vulnerability } \\
\text { Type }\end{array}$ \\
\hline phpns 2.1.1alpha & 4224 & 30 & 40 & 13 & Authz/Authc \\
\hline DCPPortal 5.1.44 & 89074 & 362 & 308 & 34 & $\begin{array}{l}\text { param tampering } \\
\text { Authc/Authz }\end{array}$ \\
\hline myBloggie 2.1.3 & 6261 & 59 & 24 & 5 & $\begin{array}{l}\text { param tampering } \\
\text { Authc/Authz }\end{array}$ \\
\hline miniBloggie 1.1 & 1283 & 11 & 5 & 2 & Authc/Authz \\
\hline SCARF 1.0 & 978 & 19 & 13 & 7 & Authc/Authz \\
\hline SnipeGallery & $9.1 \mathrm{k}$ & 37 & 25 & 3 & param tampering \\
\hline SPHPBlog & $26.5 \mathrm{k}$ & 125 & 122 & 11 & param tampering \\
\hline PHPNews & $6.4 \mathrm{k}$ & 20 & 57 & 6 & param tampering \\
\hline Landshop & $15.4 \mathrm{k}$ & 88 & 541 & 9 & param tampering \\
\hline
\end{tabular}

Path enumeration in the presence of loops Path enumeration is the most crucial component of our approach. It is used by both for patch placement and path profiling algorithms. Therefore the precision of path enumerates affects the precision of our tool. However, with static analysis of PHP code and limitations in the libraries we use, there are some cases where enumerating the paths statically is a challenge. Currently our path enumeration approach treats loops similar to if-statements and therefore each loop is treated in two different branches: 1) it is not going to be executed and 2) it is going to be executed once.

\section{Evaluation}

LOGICPATCHER, designed for patching PHP web applications, is implemented in Java in about $1.5 \mathrm{~K}$ lines of code. We use opensource tools and libraries (TAPS [8] and Pixy [13]) to get the control flow graphs for PHP applications. The experiments described in this section were performed on a MacBook Pro $(2.4 \mathrm{GHz}$ Intel, 4.0 GB RAM).

We examine 9 open-source PHP applications which are summarized in Table 2. The test suite was picked from reported logic vulnerabilities $[15,18,19]$ in PHP web applications from previous research studies, which also gave missing conditions and path locations. The results of our evaluation are categorized into three subsections: (1) the generated patches by LOGICPATCHER and sample patch and patch locations suggested by our tool, (2) the precision of LOGICPATCHER on generation and placement of the patches, and (3) scalability of our approach.

\subsection{Candidate Patch Locations}

myBloggie is a simple blogging application which lets users add, delete and update blog posts to/from a database. The application has two types of security issues: access control and parameter tampering vulnerabilities. There are six privilege escalation problems in myBloggie, which allow an unauthenticated user to delete and update blog posts. LOGICPATCHER generated a patch which checks the validity of the session variables and the level of the user suserid [' level']. The patch includes termination instructions if the security check is not met.

miniBloggie is a blogging software with standard features such as adding, deleting, and updating blog posts and comments. This application has one vertical privilege escalation(i.e., access 
to privileges in higher role) vulnerability. LOGICPATCHER fixed miniBloggie by adding the following constraint:

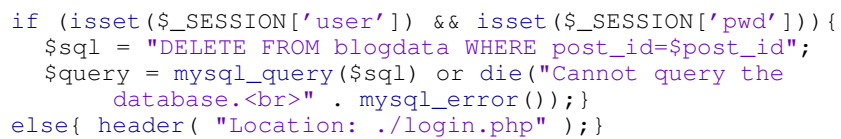

Listing 15: Patch generated for del.php, miniBloggie

SCARF is an open-source conference management software. This application is vulnerable to both vertical and horizontal privilege escalation(same role different user). The vertical escalation vulnerability is caused by lack of authorization checks in certain files. For example, in generaloptions.php the check for administrative role is omitted which lets other users to gain access to operations in this file. Our tool generated the following patch for the file, and placed the patch before the first sensitive operation (DB query) in the file.

if (\$_SESSION ['privilege' $]==$ 'admin') $\{\ldots\}$ else $\{$ exit ()$;\}$

Listing 16: Patch generated for generaloptions.php, SCARF

Several horizontal escalation vulnerabilities found in SCARF are caused by use of \$_GET['session_id'] instead of \$_SESSION [' user_id']. This vulnerability happens at the query location in the access parameters used in the WHERE clause. Therefore, for the database columns which were identified to hold the \$_SESSION [ ' user_id' ], LOGICPATCHER augmented the WHERE clause in the vulnerable queries with the necessary constraints on the column values. For the WHERE clauses in which the column was present but the value was set to \$_GET['session_id' ], LOGICPATCHER replaced the value with \$_SESSION ['user_id'] .

DCPPortal is an open-source content management system which is vulnerable to both vertical and horizontal privilege escalation. The reason for vertical privilege escalation vulnerabilities is the use of cookies from untrusted sources. LOGICPATCHER suggested using session variables instead of cookies, preserving a one-to-one correspondence between the cookie and the session variables (username, role, permissions, etc). We also generated the program slices with necessary computations for each of the new session variables. For example, for checking the admin role we added:

session_start ();

if (\$_SESSION"dcp5_member_id" $]=5)\{\ldots\}$

\section{Listing 17: Patch generated for DCPPortal}

SnipeGallery a photo album application, allows users to arrange albums hierarchically by selecting a parent category for each new album from a drop down list. By selecting a value not in that list, the new album becomes invisible. LOGICPATCHER generated the patch to check for the availability of the values in the list, before performing any sensitive operations, preventing the vulnerability.

SPHPBlog is a blog system. It uses files to store blog posts, comments, rating, etc. This application is vulnerable to parameter tampering as it does not check if the user selects values from the dropdown list, or if it is an arbitrary value. Entered values are stored in various files, which is a security threat to the server LOGICPATCHER generated the patch to check the values for the drop-down lists.

PHPNews is a news management software and is vulnerable to parameter tampering attacks. In admin.php, the application allows administrators to modify certain files through a form which contains name of the file as a hidden field. The server-side code fails to validate that the file name is not tampered and as a result attackers can update existing files, create arbitrary files and / or corrupt files of other applications deployed on the same web server.
Landshop is a real estate application which is vulnerable to both parameter tampering and horizontal privilege escalation attacks. This application includes a form with a hidden field not relevant to that form. When the value of this field is set to the ID of an existing listing (which are displayed prominently on the site), that listing is deleted from the application whether the user is the owner or not. LOGICPATCHER patched this application by 1) augmenting the WHERE clause query to include the ownership constraints and 2) generate the program slice to create the ownership value.

phpns, In phpns, application, we have a case of nondisjoint paths because of the existence of a generic function delete_item. This function is used to delete rows from any table in the DB and the table (the resource) name depends on the path leading to this function. That is, in manage.php and article.php we have:

delete_item('articles', \$items_f);

Listing 18: manage.php, phpns

delete_item('comments', \$items_f);

Listing 19: article.php before the patch, phpns,

However, only one of these execution paths is vulnerable to vertical privilege escalation, and therefore only one of these function calls should be patched by an authorization check. In this case, we use path profiling to restrict the path which deletes comments (Listing 19) to authenticated users. The generated candidate patch is shown in Listing 20:

if(\$_SESSION['username']) \{//added security check, checks
if the a valid username exists in the session
delete_item('comments', \$items_f);

Listing 20: article.php after the patch, phpns,

\subsection{Effectiveness}

We evaluated the effectiveness of our tool by manually inspecting the generated patches. We also compare the newer versions of applications in our test suite (if available) with our the patches. There are two aspects to the effectiveness of LOGICPATCHER: correctness of the patches and optimizing patch placement.

Correctness The correctness of the patching depends on: 1) the correctness of the generated patch, and 2) the correctness of the scope and location of the patch. We have confirmed that LoGICPATCHER could correctly patch 27 of 29 vulnerable files in 9 web applications in our test suite. As discussed in the Limitations subsection in Section 3, cascading sinks are one of the reasons LoGICPATCHER might generate incorrect or inconsistent patches. The following example in Scarf application shows the code before and after the patch:

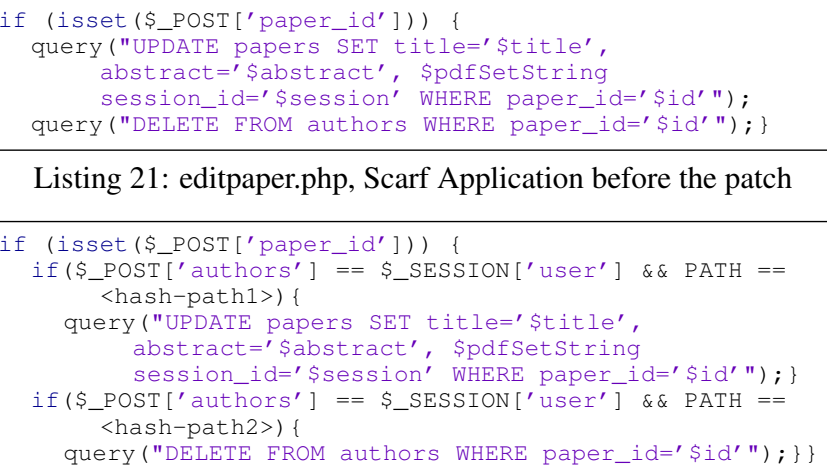

Listing 21: editpaper.php, Scarf Application before the patch

Listing 22: editpaper.php, Scarf Application after applying the candidate patch 
Table 3: Application Complexity

\begin{tabular}{|l|l|l|l|l|}
\hline Application & \# of paths & $\begin{array}{l}\text { vulnerable } \\
\text { file(s) }\end{array}$ & $\begin{array}{l}\text { Entry Point } \\
\text { Locs }\end{array}$ & $\begin{array}{l}\text { Analysis } \\
\text { time (s) }\end{array}$ \\
\hline phpns 2.1.1alpha & 709 & 1 & 21 & 3759 \\
\hline DCPPortal 5.1.44 & 588 & 12 & 210 & 2452 \\
\hline myBloggie 2.1.3 & 98 & 7 & 45 & 1620 \\
\hline miniBloggie 1.1 & 14 & 1 & 6 & 732 \\
\hline SCARF 1.0 & 86 & 3 & 16 & 250 \\
\hline SnipeGallery & 530 & 1 & 32 & 2415 \\
\hline SPHPBlog & 251 & 1 & 76 & 5613 \\
\hline PHPNews & 36 & 2 & 10 & 179 \\
\hline Landshop & 362 & 1 & 44 & 1205 \\
\hline
\end{tabular}

As it is shown in Listing 22, because two different sinks exist in the same path and they are not disjoint, the path profiling procedure will create two different hash values for the functions, and each sink is wrapped in a different set of conditions. However, these two queries should come together to preserve the consistency of the DB data.

Optimization The second aspect of the effectiveness of our tool is to check whether the patch placement was optimal. For the same missing condition $C$, our tool should be able to find the best location to inject $C$ so that multiple vulnerabilities are prevented. Currently, the path enumeration and path profiling procedures use the information about vulnerable paths and missing conditions one at a time. If the same missing condition causes multiple vulnerabilities and paths are disjoint from correct paths, optimal patches are generated. However, if any interference exists in the code, then the path profiling procedure may suggest several patch locations for multiple vulnerabilities for the same cause. We plan to address optimizing of multiple patch placement in future work.

\subsection{Scalability}

We evaluate LOGICPATCHER on variety of web applications with sizes ranging from $1 \mathrm{~K}$ to $90 \mathrm{~K}$. as shown in Columns $2-3$ in Table 2 show the size and number of PHP files in the applications, and column 4 gives an estimation of the number of sink locations (query locations, file operations, etc) in the source-code of the applications. Column 5 in Table 2 shows number of resources to be analyzed. By resources, we mean the number of DB queries in case the vulnerabilities are related to DB operations and number of different files when the sink type is file operation.

Table 3 shows statistics about the complexity of each application. In particular, it shows the total number of paths (column 2), the number of files which are vulnerable and need to be patched (column 3). Column 4 shows the number of program entry points, which together with the number of paths affect the patch placement process. That is, if the number of paths increase while the number of entry points remain the same, the number of disjoint paths would decrease. This in turn will increase the analysis times which are shown in column 5 of Table 3 . About $90 \%$ of the analysis time is spent on path enumeration which is used by the patch placement module. This analysis is only needed once and it does not add any overhead to the application execution at run-time.

\section{Related Work}

The problem of finding logic errors in software has been studied originally in the context of e-commerce business validation logic. Prior work towards detecting computational logic errors include model checking [9], modeling correct payment logics combined with static analysis [20], and invariant generation and blackbox testing [16]. Other works in this context look at finding inconsistencies using server and client side validation, by comparing the checks performed by client-side JavaScript as an implied specification to check for server-side vulnerabilities $[6,7,3]$.
In terms of finding security logic errors, RoleCast [18] is one of first works for web applications, using patterns to model authorization requirements and check if any sensitive operations are performed after authorization. Relatedly, MACE and AutoISES [15, 22] look at conditions along program paths to detect inconsistencies. MACE [15] employs a precise and fine-grained authorization model that is supported by user annotations, comparing the consistency of checking conditions across different requests to the same resources along different code paths, giving it the ability to detect a larger class of vulnerabilities. AutoISES [22] can detect bugs in standard $\mathrm{C}$ libraries through mining for common security-related patterns and identifying deviations from these as vulnerabilities. Srivastava et al. [21] detect security vulnerabilities through comparing different implementations of the same API using security policies as inputs. Any inconsistency between the security policy and any of the implementations or between different implementations are reported as errors.

The problem of fixing security errors has received less attention. Ganapathy et al. [11] study correct enforcement of authorization rules in legacy applications, such as X SERVER using a reference monitor for authorization policies. Also, static analysis tools have been used to generate patches in vulnerable software automatically, including repair by generating invariants from correct executions statically [12], placement of sanitization functions by taint analysis [14], and searching for violations in pre-defined patterns [17], requiring to a few lines of edits in the source code, or restricted to specific code transformations within a single procedure [4].

The work closest in spirit to LogicPatcher is FixMeUp[19], for fixing access control bugs in web applications due to incorrect conditions. At a high level, LOGICPATCHER is tackling a problem of broader scope, that of fixing logic vulnerabilities caused by missing or inconsistent checks, with minimal guidelines about the vulnerability. FixMeUp requires an explicit and correct high-level specification of access control checks to generate a low level policy specification and a program transformation template, computed using interprocedural backward slicing similar to LOGICPATCHER. In our work, the focus is on correct patch placement in existing code, different from their statement matching and replacement semantics. Also we do not require explicit roles or a specification of correct access control check in advance. LOGICPATCHER works using only correct conditions and path identifiers as input, and optimizes patch placement directly.

\section{Conclusions}

We present LOGICPATCHER, a tool for automatically patching application inconsistency vulnerabilities in web applications. Though LOGICPATCHER is best effort, and works without explicit functional or policy specifications, we showed that we were able to generate near-optimal patches and fix important vulnerabilities on 9 open source PHP web applications that were previously studied in literature from the point of view of vulnerability detection, inspite of inherent limitations such as cascading sinks. Verifying the correctness of these patches by hand demonstrates that LOGICPATCHER works well in identifying the correct scope and placing the patch in optimal code locations.

\section{Acknowledgement}

The authors would like to thank the anonymous reviewers for their constructive comments. This material is based upon work supported in part by the National Science Foundation under Grant Nos. NSF-CNS 1065537, NSF-CNS 1514142, NSF-DGE 1069311, DARPA FA8750-12-C-0166 and DARPA FAFA8650-15-C-7561. Any opinions, findings, and conclusions or recommendations expressed in this material are those of the author(s) and do not necessarily reflect the views of the National Science Foundation or the U.S. government. 


\section{References}

[1] Sans critical security controls for effective cyber defense, 2015.

[2] Aho, A. V., Sethi, R., And Ullman, J. D. Compilers: Principles, Techniques, and Tools. Addison-Wesley Longman Publishing Co., Inc., Boston, MA, USA, 1986.

[3] Alkhalaf, M., Choudhary, S. R., Fazzini, M., Bultan, T., Orso, A., AND Kruegel, C. Viewpoints: Differential string analysis for discovering client- and server-side input validation inconsistencies. In Proceedings of the 2012 International Symposium on Software Testing and Analysis (New York, NY, USA, 2012), ISSTA 2012, ACM, pp. 56-66.

[4] Andersen, J., And Lawall, J. L. Generic patch inference. In 23rd IEEE/ACM International Conference on Automated Software Engineering ASE 08 (2008).

[5] BALL, T., AND LARUS, J. R. Efficient path profiling. In Proceedings of the 29th Annual ACM/IEEE International Symposium on Microarchitecture (Washington, DC, USA, 1996), MICRO 29, IEEE Computer Society, pp. 46-57.

[6] Bisht, P., Hinrichs, T., Skrupsky, N., Bobrowicz, R., AND Venkatakrishnan, V. N. Notamper: Automatic blackbox detection of parameter tampering opportunities in web applications. In Proceedings of the 17th ACM Conference on Computer and Communications Security (New York, NY, USA, 2010), CCS '10, ACM, pp. 607-618.

[7] Bisht, P., HinRICHS, T., SKRUPSKY, N., AND VENKATAKRISHNAN, V. N. Waptec: Whitebox analysis of web applications for parameter tampering exploit construction. In Proceedings of the 18th ACM Conference on Computer and Communications Security (New York, NY, USA, 2011), CCS '11, ACM, pp. 575-586.

[8] Bisht, P., Sistla, A. P., And Venkatakrishnan, V. N. Taps: Automatically preparing safe sql queries. In Proceedings of the 17th ACM Conference on Computer and Communications Security (New York, NY, USA, 2010), CCS '10, ACM, pp. 645-647.

[9] Felmetsger, V., Cavedon, L., Kruegel, C., AND VIGNA, G. Toward automated detection of logic vulnerabilities in web applications. In Proceedings of the 19th USENIX Conference on Security (Berkeley, CA, USA, 2010), USENIX Security'10, USENIX Association, pp. 10-10.

[10] Ganapathy, V., Jaeger, T., And Jha, S. Automatic placement of authorization hooks in the Linux Security Modules framework. In Proceedings of the 12th ACM Conference on Computer and Communications Security (Nov. 2005), pp. 330-339.

[11] Ganapathy, V., JaEger, T., AND JHA, S. Retrofitting legacy code for authorization policy enforcement. 2012 IEEE Symposium on Security and Privacy 0 (2006), 214-229.

[12] Jin, G., Song, L., Zhang, W., Lu, S., AND Liblit, B. Automated atomicity-violation fixing. In Proceedings of the 32Nd ACM SIGPLAN Conference on Programming Language Design and Implementation (2011).

[13] Jovanovic, N., Kruegel, C., And Kirda, E. Pixy: A static analysis tool for detecting web application vulnerabilities (short paper). In Proceedings of the 2006 IEEE Symposium on Security and Privacy (Washington, DC, USA, 2006), SP '06, IEEE Computer Society, pp. 258-263.

[14] Livshits, B., AND CHONG, S. Towards fully automatic placement of security sanitizers and declassifiers. In Proceedings of the 40th Annual ACM SIGPLAN-SIGACT Symposium on Principles of Programming Languages (New York, NY, USA, 2013), POPL '13, ACM, pp. 385-398.

[15] Monshizadeh, M., NAldurg, P., AND
Venkatakrishnan, V. N. Mace: Detecting privilege escalation vulnerabilities in web applications. In Proceedings of the 2014 ACM SIGSAC Conference on Computer and Communications Security (New York, NY, USA, 2014), CCS '14, ACM, pp. 690-701.

[16] Pellegrino, G., And Balzarotti, D. Toward black-box detection of logic flaws in web applications. In NDSS 2014, Network and Distributed System Security Symposium, 23-26 February 2014, San Diego, USA (2014).

[17] Perkins, J. H., Kim, S., Larsen, S., Amarasinghe, S., Bachrach, J., Carbin, M., Pacheco, C., Sherwood, F., Sidiroglou, S., Sullivan, G., Wong, W.-F., Zibin, Y., ERnst, M. D., AND RinARD, M. Automatically patching errors in deployed software. In Proceedings of the ACM SIGOPS 22Nd Symposium on Operating Systems Principles SOSP 09 (2009).

[18] Son, S., McKinley, K. S., And Shmatikov, V. Rolecast: finding missing security checks when you do not know what checks are. In Proceedings of the 2011 ACM international conference on Object oriented programming systems languages and applications (New York, NY, USA, 2011), OOPSLA '11, ACM, pp. 1069-1084.

[19] Son, S., Mckinley, K. S., And Shmatikov, V. Fix me up: Repairing access-control bugs in web applications. In In Network and Distributed System Security Symposium (2013).

[20] Son, S., And Shmatikov, V. Saferphp: Finding semantic vulnerabilities in php applications. In ACM PLAS (2011).

[21] Srivastava, V., Bond, M. D., MCKinley, K. S., AND Shmatikov, V. A security policy oracle: Detecting security holes using multiple api implementations. In Proceedings of the 32Nd ACM SIGPLAN Conference on Programming Language Design and Implementation (2011).

[22] Tan, L., Zhang, X., Ma, X., Xiong, W., And Zhou, Y. utoises: Automatically inferring security specifications and detecting violations. In Proceedings of the 17th Usenix Security Symposium (2008).

[23] Vijayakumar, H., Ge, X., Payer, M., And Jaeger, T. Jigsaw: Protecting resource access by inferring programmer expectations. In Proceedings of the 23rd USENIX Conference on Security Symposium (Berkeley, CA, USA, 2014), SEC'14, USENIX Association, pp. 973-988. 


\section{APPENDIX}

\section{A. Error Handling}

As described in Section 4 LogicPatcher mines the security exception handling information from source-code. This preprocessing step helps the user of LOGICPATCHER to consistently handle exceptions throughout the whole application. LoGICPATCHER users can decide which of the mined instructions should be included in $E$. Table 4 shows some sample handling methods used by different applications.

Table 4: Mined Security Exceptions by LogicPatcher

\begin{tabular}{|l|l|}
\hline Application & Security Exception Handling Method \\
\hline DCPPortal & $\begin{array}{l}\text { no else branch } \\
\text { Termination } \\
\text { Redirect to Login page }\end{array}$ \\
\hline SCARF & $\begin{array}{l}\text { Termination } \\
\text { Redirect to Login page }\end{array}$ \\
\hline SPHPBlog & Redirect to Login page \\
\hline SnipeGallery & Redirect to Login page \\
\hline PHPNews & $\begin{array}{l}\text { Termination } \\
\text { Redirect to Login page }\end{array}$ \\
\hline MiniBloggie & $\begin{array}{l}\text { Termination } \\
\text { Redirect to Login page }\end{array}$ \\
\hline MyBloggie & Termination \\
\hline PHPNS & $\begin{array}{l}\text { Termination } \\
\text { Redirect to Login page }\end{array}$ \\
\hline Landshop & Termination \\
\hline
\end{tabular}

